
Revue
de /histoire des religions
Revue de l'histoire des religions
$3 \mid 2009$
La culture gallicane. Références et modèles (droit, ecclésiologie, histoire)

\title{
Jacques Auguste de Thou (1553-1617) : une rupture intellectuelle, politique et sociale
}

Jacques Auguste de Thou (1553-1617): Intellectual, Political and Social Rupture

\section{Robert Descimon}

\section{OpenEdition}

\section{Journals}

Édition électronique

URL : http://journals.openedition.org/rhr/7282

DOI : $10.4000 /$ rhr.7282

ISSN : 2105-2573

Éditeur

Armand Colin

\section{Édition imprimée}

Date de publication : 1 juillet 2009

Pagination : 485-495

ISBN : 978-2200-92591-8

ISSN : 0035-1423

Référence électronique

Robert Descimon, « Jacques Auguste de Thou (1553-1617): une rupture intellectuelle, politique et sociale », Revue de l'histoire des religions [En ligne], 3 | 2009, mis en ligne le 01 juillet 2012, consulté le 01 mai 2019. URL : http://journals.openedition.org/rhr/7282 ; DOI : 10.4000/rhr.7282 


\section{ROBERT DESCIMON}

Centre de recherches histEriques, Paris

(ÉcEle des Hautes Études en Sciences SEciales -

Centre NatiEnal de la Recherche Scientifique)

\section{Jacques Auguste de Thou (1553-1617) : une rupture intellectuelle, politique et sociale}

Le président de Thou a compté parmi les créateurs de la nouvelle configuration intellectuelle qui domina tout le XVII siècle. Tout en se situant dans la tradition des magistrats gallicans, il s'inscrivit dans un mouvement de rupture. Socialement, il rompit les liens de son lignage avec l'ancien patriciat municipal parisien et devint ainsi un prototype des nobles de robe par mimétisme culturel avec la gentilhommerie dite d'épée. Surtout, son cuvre contribua à congédier les savoirs majeurs dont la Renaissance avait hérité : la théologie dogmatique et la science du droit. Le prestige de sa monumentale Histoire, écrite en latin, fonde un espace littéraire européen : sans elle, la littérature n'aurait pu conquérir les pouvoirs qui seront les siens sous le ministère de Richelieu, ni la monarchie la pratique absolutiste de son exercice.

\section{Jacques Auguste de Thou (1553-1617) : Intellectual, Political and Social Rupture}

The président de Thou was among the creators of the new intellectual configuration that dominated the entire $17^{\text {th }} C$. Even as he situated himself within the tradition of the Gallican magistrates, he was part of a movement of rupture from it. Socially, he broke off hereditary ties with the old municipal patrician class of Paris and became a prototype of the nobleman of the gown through cultural imitation of the nobles of the sword. Most important, his work contributed to the dismissal of the major fields of knowledge that the Renaissance had inherited: dogmatic theology and legal science. The prestige of his monumental Latin Histoire opened up a new European literary space. Without this work, literature could not have attained the power that it enjoyed under Richelieu, nor could the monarchy have attained the absolutist practice of its own power. 
Avec des fortunes diverses, le président Jacques Auguste de Thou se fit le chef du parti dit gallican jusqu'à sa mort. Il convient, bien sûr, de distinguer le gallicanisme comme courant de pensée (on n'ose dire encore " politique ») et l'Église gallicane comme institution dotée de libertés dont peu de Français, laïcs ou clercs, mettaient en cause à l'époque le bien-fondé. De Thou s'inscrivait donc comme chrétien gallican dans une continuité partisane dont les racines sont à chercher dans la Pragmatique Sanction ${ }^{1}$. Dans les conditions propres au règne de Henri IV, il se construisit une personnalité intellectuelle par une série de ruptures que manifeste hautement son œuvre. Il fut incontestablement un précurseur, inscrit dans une configuration intellectuelle qu'il contribua à fonder, celle de la « république des lettres » $\mathrm{du} \mathrm{XVII}^{\mathrm{e}}$ siècle $^{2}$, ce qui supposait une prise de distance avec le mouvement humaniste né du XvI ${ }^{\mathrm{e}}$. De Thou contribua ainsi à congédier une époque. Je voudrais étayer cette assertion autour de trois arguments.

\section{UNE EXPÉRIENCE SOCIALE ET POLITIQUE PERSONNELLE}

1. De Thou rompit d'abord précocement avec un certain passé de son lignage. Il répudie un des héritages que lui avaient transmis son père Christophe, le premier président, et son oncle Augustin, l'avocat général devenu lui-même président: une implantation municipale qui situait la famille depuis son installation à Paris au sein de l'oligarchie bourgeoise qui contrôlait l'Hôtel de Ville. De Thou travailla son appartenance sociale en se rapprochant de la

1. Jotham Parsons, The Church in the Republic. Gallicanism and Political Ideology in Renaissance France, Washington, The Catholic University of America Press, 2004. Roger Zuber, « Cléricature intellectuelle et cléricature politique : le cas des érudits gallicans (1580-1620) », dans Travaux de linguistique et de littérature, Strasbourg, Centre de philologie et de littérature romanes, XXI/2, 1983, p. 121-134.

2. De Thou s'entoura d'une équipe de savants à Paris et constitua un réseau de correspondants en Europe qui partageaient jusqu'à un certain point ses conceptions religieuses et politiques. La notion de « république des lettres » est problématique (pour une description: Hans Bots et Françoise Waquet, La République des lettres, Paris, Belin, 1997). Jérôme Delatour, "Le cabinet des frères Dupuy », Revue d'histoire des facultés de droit et de la science juridique, 25-26, 2005-2006, p. $157-200$. 
bonne noblesse à laquelle il s'allia par ses deux mariages ${ }^{3}$. Il contribua de façon pertinente à fonder les prétentions de la haute robe à se constituer en «noblesse», une noblesse certes différente de la noblesse d'épée, mais une noblesse à part entière ${ }^{4}$ participant de valeurs nobiliaires communes : la robe et l'épée devenaient idéalement deux «partis » qui s'offraient aux jeunes gens bien nés sans hiérarchisation spéciale entre les deux professions qui servaient le roi en deux de ses fonctions essentielles.

2. Cette mue sociale avait aussi un fondement politique : la détestation des pratiques civiques de participation urbaine qui faisaient que la maison commune de la place de Grève était gérée, certes per gradus, selon une échelle différentielle des dignités par l'ensemble des participants aux privilèges de bourgeoisie, y compris, pour une très faible part, en raison du déficit d'honneur attaché à leur condition, par les artisans dits «mécaniques $»^{5}$. Cette hostilité de De Thou provenait évidemment de l'interprétation qu'il donnait aux guerres civiles d'après son expérience. À ses yeux, la bourgeoisie parisienne avait été gagnée par l'ultra-catholicisme en vertu même de cette structure politique dont l'expression confraternelle était le versant religieux, certes, mais aussi social et politique. La Ligue peut s'interpréter, dans les termes du temps, comme une tentative pour former une confrérie centrale à l'échelle de toute la communauté citadine ${ }^{6}$. Dans le programme politique de De Thou, il n'y

3. Robert Descimon, "Les de Thou au miroir des archives notariales du $\mathrm{XVI}^{\mathrm{e}}$ siècle. Les chemins de la haute robe ", dans Jacques-Auguste de Thou. Écritures et condition robine, Cahiers V. L. Saulnier 24, Paris, Presses de l'Université de Paris-Sorbonne, 2007, p. 13-35.

4. Albert Cremer, « La genèse de la notion de noblesse de robe », Revue d'histoire moderne et contemporaine, 46/1, 1999, p. 22-38.

5. Pour une description du fonctionnement social de la municipalité parisienne, Robert Descimon, «Le corps de ville et les élections échevinales à Paris aux Xvi ${ }^{\mathrm{e}}$ et $\mathrm{XVII}^{\mathrm{e}}$ siècles. Codification coutumière et pratiques sociales », Histoire économie et société, 13/3, 1994, numéro Lectures de la ville (XV-XX siècle), Jacques Bottin et Alain Cabantous (dir.), p. 507-530.

6. Christopher W. Stocker, « The Confraternity of the Holy Name of Jesus. Conflict and Renewal in the Sainte Union in 1590 », dans Confraternities and Catholic Reform in Italy, France, and Spain, John Patrick Donnelly et Michael W. Maher (éd.), Kirksville, Sixteenth Century Essays and Studies XLIV, 1999, p. 155-189, et Ann W. Ramsey, «From Ontology to Religious Experience : Civic and Sacred Immanence in the Holy Sacrament Confraternities of Paris during the Catholic League », ibid., p. 135-153. Robert Descimon, « La Ligue à Paris (15851594) : une révision », Annales (Économies Sociétés, Civilisations), 37/1, 1982, p. $72-111$. 
avait rien de pire, car il voyait là l'origine des massacres perpétrés contre les protestants et de la révolte contre l'autorité royale?

Ces deux projets poussaient de Thou à adopter une stratégie de distinction tendant à le séparer et à séparer sa famille de son terreau bourgeois d'origine. La culture était le moyen privilégié de cette opération de sécession.

\section{UNE CULTURE DE SÉGRÉGATION}

1. La Vita constitue une mise en scène remarquable d'une existence d'exception fondée sur le savoir et le raffinement ${ }^{8}$. L'éducation humaniste produite par la culture gréco-romaine et une pratique active de la langue latine, l'écriture poétique, la passion des livres et des manuscrits comme objets, la pratique du voyage culturel, la fréquentation des meilleurs esprits, le goût pour les arts, en particulier le dessin, où de Thou dit s'être complu, le commerce des grands et l'accomplissement auprès d'eux de missions quasi diplomatiques, tout cela contribue à brosser le tableau d'une âme d'élite détachée des préoccupations d'un vain peuple, mais aussi des pratiques professionnelles propres à la magistrature, sur lesquelles la Vita, comme les poésies, fait silence ou porte une appréciation négative. Assurément, même dans la robe et au parlement, peu de magistrats pouvaient revendiquer des horizons culturels aussi larges que le faisait de Thou avec quelque ostentation. De ce point de vue la Vita peut être lue en parallèle avec le Discours de la vie de son beau-frère, le premier président Achille de Harlay, discours qui insiste avant tout sur la figure du «heros togatus $»^{9}$ dont l'essence est à rechercher

7. D'où son hostilité contre les cloches, symboles de la communauté catholique. Ingrid A. R. De Smet, «Contre les cloches : autour de neuf épigrammes inédites de Jacques-Auguste de Thou ", Bibliothèque d'humanisme et Renaissance, LXVI/1, 2004, p. 91-110 (le vers Vis sitis potius civilis classica bellis ne signifie pas 'vous feriez mieux d'être le signal de la guerre civile', mais 'vous avez mieux à faire que d'être le signal de la guerre civile').

8. Jacques-Auguste de Thou, I. Aug. Thuani vita, introduction, établissement du texte, traduction et notes par Anne Teissier-Ensminger, Paris, Champion, 2007. Ingrid A. R. De Smet, Thuanus : The Making of Jacques-Auguste de Thou, Genève, Droz, 2006, p. 147-200.

9. Jacques de La Valée [aumônier du roi et « jadis domestique dudit seigneur de Harlay »], Discours sur la vie, actions et mort de très illustre seigneur, Messire Achilles de Harlay, en son vivant conseiller du roy en ses Conseils d'Estat 
dans la dignité et l'autorité du juge représentant de la figure royale et donc sur l'expertise politique fondée par la science du droit.

2. La latinité de l'écriture de De Thou remplit une fonction essentielle. Il n'écrivait pas pour tous, mais seulement pour les savants et les puissants, français ou non ${ }^{10}$. On mesure ici la distance qui sépare sa stratégie d'écriture, visant à une réception élitiste, de celle de Guillaume Du Vair. Ce dernier choisit le français, sans doute par insuffisance linguistique, mais aussi par un choix plus positif: ouvrant les questions brûlantes du statut de l'éloquence dans un régime monarchique, de la pertinence de la philosophie stoïcienne comme défense contre les entraînements de la guerre civile, et de l'expression d'une foi catholique affirmée sous la forme d'une littérature spirituelle, il visait une aire de réception très large en France, dépassant le cercle étroit des lettrés ${ }^{11}$. De ce fait, il établissait d'ailleurs une complicité moins nette que ne le faisait de Thou avec l'intelligentsia européenne. Le parallèle de De Thou et de Du Vair est d'autant plus révélateur que leurs intérêts littéraires les avaient rapprochés dans les premières années de leur vie d'homme, par exemple dans le commentaire du Livre de Job ou des Psaumes ${ }^{12}$.

3. La veine poétique néo-latine de De Thou s'inscrit certes dans une tradition, illustrée en particulier par le chancelier Michel de L'Hospital. Mais elle manifeste une proximité plus marquée avec la poésie de Cour et une affirmation assez nette de préjugés aristocratiques. Son immense poème Hieracosophioy [Hieracosophiou], sive De venatione per accipitres libri duo (tres) fut entrepris sous l'influence du petit-fils du chancelier, Michel Hurault, un magistrat, certes, et de conviction calviniste, mais aussi un homme qui

et privé, premier president du sénat de Paris et comte de Beaumont en Gatinois pour servir d'exemple à ceux qui pour l'advenir voudront saintement administrer la justice, Paris, Corrozet, 1616, réédité par L. Cimber et F. Danjou, Archives curieuses de l'histoire de France, Paris, Beauvais, 1837, t. XV, p. 423-457. JeanMarc Chatelain, "'Heros togatus' : culture cicéronienne et gloire de la robe dans la France d'Henri IV », Journal des Savants, 1991, p. 263-287.

10. Sur l'œuvre de De Thou : Samuel Kinser, The Works of Jacques-Auguste de Thou, La Haye, Nijhoff, 1966.

11. Voir les études réunies sous le titre Guillaume Du Vair. Parlementaire et écrivain (1556-1621), par Bruno Petey-Girard et Alexandre Tarrête, Genève, Droz, 2005.

12. Ingrid A. R. De Smet, « La poésie sur le fumier. La figure de Job à l'époque des guerres de Religion », dans Jacques-Auguste de Thou..., op. cit., p. 89-106. Guillaume Du Vair, Premières æeuvres de piété, éd. Bruno Petey-Girard, Paris, H. Champion, 2002, p. 7-23. 
se piquait de gentilhommerie, se passionnait pour la chasse et mourut, dit-on non sans malveillance, de ne pas avoir vu ses capacités militaires suffisamment reconnues par Henri IV. De Thou travailla quatre ans à ce poème ${ }^{13}$. L'angoisse que de Thou nourrissait à l'égard de cette œuvre ambitieuse ne tenait vraisemblablement pas seulement aux difficultés de la prosodie latine, mais au sujet lui-même : la fauconnerie, passe-temps emblématique de la noblesse, forme de chasse la plus technique et la plus aristocratique et, à ce titre, critère de distinction de première force. Il y avait là plus encore peut-être : la fauconnerie symbolisait un mode d'appropriation de l'espace caractéristique des conceptions du pouvoir nobiliaire ${ }^{14}$. Le jeune de Thou, en s'intéressant savamment à la question, même si sa pratique n'était pas très suivie, proclamait par là son appartenance à la vraie noblesse, ce qui supposait d'adopter les intérêts culturels des gens d'épée. Mais, en même temps, il se classait parmi les savants à tel degré qu'aucun noble n'était capable de rivaliser avec lui. De Thou réussissait ainsi à sous-entendre à la fois son appartenance à la gentilhommerie traditionnelle et sa supériorité.

\section{LA PROMOTION DE NOUVELLES FORMES DE LA CULTURE LETTRÉE}

1. Au contraire, peut-être, de ses Poésies ${ }^{15}$, on ne saurait réduire le grand œuvre de De Thou, c'est-à-dire son Histoire, à une visée tactique (le «going public» l'emportant sur le «making»). Les buts d'écriture de ce travail cyclopéen ont été suffisamment explicités par de Thou lui-même et par ses continuateurs et amis ${ }^{16}$. L'inves-

13. $1^{\text {re }}$ édition, Bordeaux, Millanges, 1582, et trois rééditions de son vivant (1584 (avec inclusion d'un troisième livre), 1587, chez Patisson à Paris, la dernière, en 1609, à Francfort).

14. Philippe Salvadori, La chasse sous l'Ancien Régime, Paris, Fayard, 1996, p. 43-45. Denis Crouzet, La nuit de la Saint-Barthélemy. Un rêve perdu de la Renaissance, Paris, Fayard, 1994, p. 295-306, « la politique racontée par l'histoire d'un roi chasseur $»$.

15. Ingrid A. R. De Smet, " Going Public : Rewriting and Self-Fashioning in the Early Poetry of Jacques-Auguste de Thou », Studies in Early Modern France, $\mathrm{n}^{\circ} 8$ (Strategic Rewriting), 2002, p. 25-42.

16. Kinser, op. cit., et De Smet, Thuanus..., op. cit. Et cette explication alla jusqu'à une réécriture de l'histoire qui se révéla pleinement à l'âge des Lumières, Alfred Soman, «The London edition of de Thou's History : a Critique of Some Well-documented Legends ", Renaissance Quaterly, n² 24, 1971, p. 1-12. C'est toute la question de la mise à l'Index de l'Historia sui temporis. 
tissement intellectuel qu'il représentait s'inscrivait dans un projet social et monarchique. Il fut couronné d'un succès entier, non seulement par la réception enthousiaste qui l'accueillit dans l'élite européenne attachée à promouvoir des paix de religion, mais aussi par la postérité. L'interprétation historique des guerres de Religion repose jusqu'à aujourd'hui sur les analyses produites par de Thou et le cabinet des Dupuy. Ce succès n'a aucun exemple historiographique. Il est sidérant et fondateur : c'est une nouvelle forme d'histoire, engagée et non objective, certes, qui est inaugurée par de Thou, une histoire protéiforme dans la mesure où elle sera travaillée par diverses herméneutiques, celles des protestants et catholiques irénistes, celles des «libertins", celles des Lumières, celles des républicains français et finalement des progressistes du monde entier jusqu'à la mondialisation ${ }^{17}$. Mais cette rupture fondatrice eut un prix que les historiens des idées ont rarement souligné.

2. La première cassure s'effectue avec la vision théologique du monde. La théologie dogmatique est congédiée comme politiquement incorrecte et la théologie mystique prend sa place auprès de certains élus qui en ont reçu la grâce ${ }^{18}$. C'est un trait commun au parti royal, surtout dans sa composante catholique, d'esquiver la question qui fâche par excellence, celle de l'eucharistie et de la présence réelle $^{19}$. Certes, l'Histoire de De Thou ne rompt pas avec une vision providentialiste, mais cette providence n'est plus globale, orientée vers la Chrétienté dans son ensemble, elle est devenue locale, inscrite dans l'élection divine du royaume de France et de son roi ${ }^{20}$. Certes, les diverses «naissances de la nation France », celle du Xv siècle,

17. Corrado Vivanti, Lotta politica e pace religiosa in Francia fra Cinque e Seicento, Turin, Einaudi, 1963, p. 292-324 (tr. fr. partielle, Guerre civile et paix religieuse dans la France d'Henri IV, Paris, Desjonquères, 2006). De cette étonnante fortune, on trouvera un témoignage frappant dans l'étude, par ailleurs critique, d'Antony Grafton, Les origines tragiques de l'érudition. Une histoire de la note en bas de page, Paris, Seuil, 1998, p. 109-116.

18. Michel de Certeau reste ici le guide irremplaçable (La fable mystique XVI ${ }^{e}$ XVII siècle, Paris, Gallimard, 1982).

19. Daniel Ménager, « Dieu et le roi », dans Frank Lestringant et Daniel Ménager (éd.), Études sur la Satyre Ménippée, Genève, Droz, 1987, p. 201-226.

20. Alexandre Tarrête, "Présages et prodiges chez Jacques-Auguste de Thou (1553-1617)», dans Hasard et Providence, XLIX ${ }^{\mathrm{e}}$ colloque international d'études humanistes, Tours, 3-9 juillet 2006, Marie-Luce Demonet (dir.), consultable sur http ://www.cesr.univ-tours.fr/publications/HasardetProvidence/fichiers/ pdf31_Tarrete.pdf. 
vue par Colette Beaune ${ }^{21}$, celle des guerres de Religion, vue par Miryam Yardenii2 ${ }^{2}$, avaient déjà exploité cette veine. Mais le nouveau contexte créé par l'avènement des Bourbons donna une consistance bien supérieure à cette vision des choses européennes. Après tout, on peut même en trouver l'écho laïcisé dans le patriotisme républicain et anti-catholique de Michelet ${ }^{23}$. La rupture avec la conception catholique, apostolique et romaine de l'histoire a été suffisamment analysée pour qu'il soit à peine besoin d'y revenir. Mais il y avait aussi là une question de bon goût, de mode même : la spiritualité affectait des formes baroques bien plus séduisantes que le lourd pédantisme de la Sorbonne, dont le doyen Jean Boucher, l'ancien curé ligueur de Saint-Benoît, donne un exemple si frappant ${ }^{24}$.

3. La seconde rupture est passée plus inaperçue. Elle a moins de portée pour l'avenir, mais plus de sens en contexte, dans le présent de l'écriture thuane. Les écrits de De Thou sont vierges de toute contribution à la «science du droit» et même à la pratique des « robes rouges », cette jurisprudence souveraine à laquelle Du Vair finit par sacrifier. Or c'était là passer à la « politique », telle que la monarchie allait la pratiquer, et renoncer à la «médiation patriarcale des juristes » qui continua à fonder la théorie et l'action des parlements jusqu'à la Révolution française ${ }^{25}$. Par une conséquence non perçue par de $\mathrm{Thou}^{26}$, le juris peritus ne pouvait plus être $i d$ est

21. Colette Beaune, Naissance de la nation France, Paris, Gallimard, 1985.

22. Myriam Yardeni, La conscience nationale en France pendant les guerres de Religion (1559-1598), Louvain, Nauwelaerts, 1971.

23. Marco Penzi, «'Damnatio memoriae' : la 'Ligue catholique française' e la storiografia, tra 'politiques', rivoluzionari, mistici e liberali », Quaderni storici, 118,2005 , p. 263-284.

24. Robert Descimon et José Javier Ruiz Ibáñez, Les ligueurs de l'exil. Le refuge catholique français après 1594, Seyssel, Champ Vallon, 2005, voir l'index pour l'action et les publications de Boucher.

25. Francesco Di Donato, L'ideologia dei robins nella Francia dei Lumi. Costituzionalismo e assolutismo nell'esperienza politico-istituzionale della magistratura di antico regime (1715-1788), Naples, Edizioni Scientifiche Italiane, 2003, et id., "Constitutionalisme et idéologie de robe. L'évolution de la théorie juridico-politique de Murard et Le Paige à Chanlaire et Mably », Annales (Histoire Sciences sociales), 52/4, 1997, p. 821-852.

26. Sur le décalage entre les positions de De Thou et leurs réceptions par la papauté et la monarchie, Robert Descimon, «Penser librement son intolérance : le président Jacques Auguste de Thou (1553-1617) et l'Épittre dédicatoire des Historiae sui temporis (1604) ", La liberté de penser. Hommage à Maurice Laugaa, François Lecercle (éd.), Poitiers, La Licorne, 2002, p. 73-86. Pour l'expression latine citée, voir Kelley, note 28. 
politicus. Car les leçons de la science du droit, en particulier des post-glossateurs, n'allaient pas clairement dans le sens du renforcement de la puissance législatrice du souverain et de son émancipation par rapport à tout contrôle humain. Le droit divin pouvait se servir des juristes, mais il avait sans doute intérêt à se passer d'eux ${ }^{27}$. On ne comprend la profondeur de cette mutation que par la considération attentive de ce qui avait fondé la pensée juridique (le mos gallicus au moins) au temps de l'Humanisme. Les recherches de Donald Kelley ont ici tracé très fermement le cadre de référence. Trois composantes pouvaient concourir à la supériorité de la science $\mathrm{du}$ droit dans le contexte renaissant : le commentaire savant du droit commun, science des jurisconsultes, science encore princesse au temps de la jeunesse de De $\mathrm{Thou}^{28}$, la logique, qu'elle soit d'Aristote ou de Ramus, liant dans un combat complice un courant conservateur et un courant novateur, enfin les conceptions mystiques qui relient par une complémentarité fondatrice le commentaire théologique et le commentaire juridique. Le jurisconsultus perfectus est une des figures éminentes de la Renaissance et le droit était «vera philosophia ». Voilà certainement des « vérités » auxquelles l'esprit critique de de Thou refusait de croire. À ce refus, deux raisons essentielles : ces diverses « sciences" s'étaient révélées impuissantes à endiguer l'esprit de guerre civile; le contexte culturel s'était sécularisé, pour ainsi dire, il entraînait des ruptures relatives, mais significatives avec les pratiques intellectuelles de la tradition (livres dorés, recueils d'emblèmes, encyclopédies portatives.... $)^{29}$, toutes techniques qu' ignoraient relativement de Thou historien, sinon ses continuateurs, les Dupuy ${ }^{30}$ et Nicolas Rigault. Le réseau des causalités

27. Fanny Cosandey et Robert Descimon, L'absolutisme en France. Histoire et historiographie, Paris, Le Seuil (collection « Points Histoire »), 2002.

28. Donald Kelley, en particulier, « Jurisconsultus perfectus : The Lawyer as Renaissance Man », Journal of the Warburg Courtauld Institutes, 51, 1988, p. 84102, repris dans The Writing of History and the Study of Law, Londres, Variorum, 1997, $\mathrm{n}^{\circ}$ XIII et « Vera Philosophia : The Philosophical Signifiance of Renaissance Jurisprudence », Journal of the History of Philosophy, XIV, 1976, p. 267-279, repris dans History, Law and the Human Sciences, Londres, Variorum, 1984, $\mathrm{n}^{\circ}$ IV.

29. Ann Blair, «Bibliothèques portables : les recueils de lieux communs dans la Renaissance tardive ", dans Le pouvoir des bibliothèques. La mémoire des livres en Occident, Marc Baratin et Christian Jacob (dir.), Paris, Albin Michel, 1996, p. 84-106. Ann Moss, Les recueils de lieux communs : méthode pour apprendre à penser à la Renaissance (1996), Genève, Droz, 2002.

30. Pierre Dupuy pratiquait cette méthode de travail pour se préparer à la profession d'avocat (Jérôme Delatour, « De l'art de plaider doctement : les notes de 
que l'on voit ici à l'œuvre formait la condition d'existence de la nouvelle « république des lettres », comme structure d'érudition, mais aussi de dialogue à l'écart des mouvements de confessionnalisation qui se développaient par ailleurs pour le plus grand bonheur des pouvoirs théologico-politiques. Le transfert de sacralité politique qui s'était opéré de l'Église sur la personne royale eut des conséquences qui ne correspondaient guère à la conciliation de la « liberté » et du « principat » telle que la rêvait l'Epître dédicatoire de l'Historia en 1604. La victoire de De Thou et des siens est donc indéniable, mais elle fut culturelle, non politique.

\section{EN GUISE DE CONCLUSION}

On peut constater la distance intellectuelle qui sépare de Thou des juristes qui restent attachés aux conceptions anciennes. Charles Loyseau, par exemple, mûrit un projet intellectuel qui allie la «science des saints" et la «scientia juris» à la fin du règne $\mathrm{d}^{\prime} H e n r i \mathrm{IV}^{31}$. Loyseau était un trop petit sire pour se mesurer à de Thou; mais il est clair que ses écrits prétendant fonder à la fois le pouvoir souverain du monarque français et l'entente cordiale de la monarchie et de l'Église, participaient d'un projet inscrit dans une tradition $\mathrm{du} \mathrm{XVII}^{\mathrm{e}}$ siècle que le parti dit dévot sut rénover, malgré son échec final. Cardin Le Bret, et sa Souveraineté du roi (1632), lui aussi contemporain de De Thou (il était né en 1559 et avait été avocat général au parlement quand De Thou présidait), donnèrent à la théorie absolutiste sa forme la plus achevée, mais cette achèvement même mécontenta Richelieu et les ministres ${ }^{32}$. La monarchie n'avait plus trop besoin de justifications juridiques, mais elle était demandeuse de propagande historique. L'échec de Le Bret le surprit sans doute. Aussi bien appartenait-il discrètement à une mou-

lecture de Pierre Dupuy, jeune avocat (1605-1606) », Bibliothèque de l'École des chartes, 153, 1995, p. 391-412).

31. Robert Descimon, « Dignité contre vénalité. L'œuvre de Charles Loyseau (1564-1627) entre science du droit et science des saints », Historische Anstöße. Festschrift für Wolfgang Reinhard zum 65. Geburtstag am 10. April 2002, éd. Peter Burschel, Mark Häberlein, Volker Reinhardt, Wolfgang E. J. Weber, Reinhard Wendt, Berlin, Akademie Verlag, 2002, p. 326-338.

32. William F. Church, Richelieu and Reason of State, Princeton, Princeton University Press, 1972. 
vance très catholique influencée par le mouvement jésuite. Cela ne déplaisait nullement au régime, qui exigeait cependant une relative séparation des genres ${ }^{33}$. C'était la fin des juristes comme penseurs politiques, ce que William Church a décrit dans un article justement réputé $^{34}$. Même si le gallicanisme de De Thou fut aussi profondément vaincu que la mouvance dévote (l'affaire Santarelli fut à ce titre le moment décisif ${ }^{35}$ ), son projet intellectuel avait triomphé. Le beau livre de Christian Jouhaud, Les pouvoirs de la littérature, a retracé les raisons cardinales de ce changement des références intellectuelles et des genres légitimes dans la France de l'époque ${ }^{36}$. Théologie dogmatique et jurisprudence savante étaient les grandes perdantes de ce contexte littéraire, leur légitimité comme modes d'intervention dans la sphère publique était radicalement mise en cause. Triomphaient des formes nouvelles, depuis les Lettres jusqu'à la tragédie, que certes Jacques Auguste de Thou n'avait pas pratiquée, mais dont il avait préparé l'avènement par la double sécularisation que ses écrits avaient promue, par rapport à l'écriture théologique et à l'écriture juridique.

descimon@ehess.fr

33. Michel de Certeau, «Politique et mystique. René d'Argenson (15961651) », Revue d'ascétique et de mystique, 39, 1963, p. 45-82.

34. William F. Church, "The Decline of the French Jurists as Political Theorists, 1660-1789», French Historical Studies, 5, 1967, p. 1-40.

35. Sylvio Hermann De Franceschi, «La genèse française du catholicisme d'État et son aboutissement au début du ministériat de Richelieu. Les catholiques zélés à l'épreuve de l'affaire Santarelli et la clôture de la controverse autour du pouvoir pontifical au temporel (1626-1627) », Annuaire-Bulletin de la Société de l'histoire de France, 2001, Paris, 2003, p. 19-63.

36. Christian Jouhaud, Les pouvoirs de la littérature, Paris, Gallimard, 2000. 